\title{
Conocimientos de los maestros y futuros maestros sobre trastornos del neurodesarrollo: una revisión teórica
}

\author{
Pilar Sanz-Cervera. Universitat de València \\ Recepción: 15.09.2018 | Aceptado: 2.10.2018 \\ Correspondencia a través de ORCID: Pilar Sanz
}

iD 0000-0001-6919-6150

Citar: Sanz-Cervera, P. (2018). Conocimientos de los maestros y futuros maestros sobre trastornos del neurodesarrollo: una revisión teórica. ReiDoCrea, 7, 288-297.

Resumen: El grado de conocimientos que los maestros poseen en materia de educación especial es un aspecto clave que condiciona el hecho de poder realizar una detección y un diagnóstico temprano, además de condicionar la calidad de la intervención que puedan ofrecer. En el caso de los trastornos del neurodesarrollo, en los últimos años se ha realizado un estudio exhaustivo sobre el nivel de conocimientos de los maestros y futuros maestros respecto al trastorno por déficit de atención con o sin hiperactividad (TDAH), pero no se han realizado tantos estudios en relación a otros dos trastornos que también poseen una prevalencia elevada, como son el trastorno del espectro autista (TEA) y las dificultades específicas del aprendizaje (DEA). Por ello, en el presente estudio se realiza una revisión teórica sobre los estudios empíricos publicados en los últimos cinco años (2014-2018) en relación a los conocimientos que poseen tanto los maestros como los futuros maestros sobre estos dos trastornos. Los resultados obtenidos evidencian un grado de conocimientos bajo, de ahí la necesidad de realizar un análisis de los planes de estudio para poder ofrecer una mejor formación dentro de un marco de escuela inclusiva.

Palabras clave: Conocimientos maestros | Trastornos del neurodesarrollo

Pre-service and in-service teachers' knowledge about neurodevelopmental disorders: a theoretical review

\begin{abstract}
The knowledge degree that teachers have in the special education area is a key aspect that determines the possibility of making an early detection and diagnosis, as well as conditioning the quality of the intervention they can offer. In the case of neurodevelopmental disorders, in recent years an exhaustive study on the level of pre-service and in-service teachers knowledge regarding attention deficit and hyperactivity disorder (ADHD) has been carried out, but not so many studies have been done in relation to two other disorders that also have a high prevalence, such as autism spectrum disorder (ASD) and learning disabilities (LD). Therefore, in the present study a theoretical review of the empirical studies published in the last five years (2014-2018) in relation to the knowledge that both pre-service and inservice teachers have about these two disorders has been made. The results obtained show a low level of knowledge; hence the need to carry out an analysis of the study plans in order to offer a better education within an inclusive school framework.
\end{abstract}

Keywords: Neurodevelopmental Disorders | Teachers' Knowledge

\section{Introducción}

La actual edición del Manual Diagnóstico y Estadístico de los Trastornos Mentales (DSM-5; APA, 2013) incluye dentro de la etiqueta de trastornos del neurodesarrollo algunos de los diagnósticos con mayor repercusión en el contexto escolar, como son: el trastorno del espectro autista (TEA), las dificultades específicas del aprendizaje (DEA) y el trastorno por déficit de atención con o sin hiperactividad (TDAH).

Las características de estos tres diagnósticos conllevan implicaciones importantes para la adaptación escolar: 
- La característica fundamental de las DEA es la dificultad específica para alcanzar uno de los objetivos fundamentales del proceso de escolarización, el aprendizaje de contenidos académicos;

- Las dificultades propias del TDAH implican que los estudiantes con este diagnóstico no sean capaces de permanecer sentados y atentos durante los períodos de tiempo que exige la escolarización;

- Y las dificultades asociadas al TEA conllevan que los estudiantes con este diagnóstico vean mermadas sus posibilidades para relacionarse e interactuar con otros niños, por lo que tienen dificultades para alcanzar otro de los objetivos de la educación, que es precisamente la socialización con los iguales.

La prevalencia de estos diagnósticos es, además, de las más elevadas, especialmente, en edad escolar. Las investigaciones señalan que la prevalencia de las DEA se sitúa entre un 5 y un 15\% (Soriano-Ferrer y Morte-Soriano, 2017), la del TDAH entre un 5,4\% (Canals, Morales-Hidalgo, Claustre-Jané, y Domènech-Llaberia, 2016) y un 6,8\% (Catalá-López et al., 2012), y la del TEA entre un 1 y un 1,55\% (MoralesHidalgo, Roigé-Castellví, Hernández-Martínez, Voltas, y Canals, 2018), por lo que es habitual encontrar aulas en educación infantil, primaria y secundaria en las que se escolaricen estudiantes con este tipo de dificultades.

Por estos motivos, la formación del profesorado en lo que respecta a los conocimientos sobre estos trastornos del neurodesarrollo es un aspecto crucial que cabe considerar. En concreto, el rol del profesorado ante estos trastornos del neurodesarrollo es relevante por distintos motivos:

1. Están en una posición idónea para identificar tempranamente síntomas o ciertas señales de alarma que puedan hacer pensar en la presencia de uno de estos diagnósticos.

2. Son los responsables directos de implementar medidas educativas: por ejemplo a través del uso de estrategias cognitivas y metacognitivas para las DEA, estrategias de autorregulación para el TDAH, o historias sociales y paneles de anticipación en el caso del TEA.

3. Son una figura de referencia para sus estudiantes, por lo que sus actitudes ante la diversidad son muy importantes, ya que son un agente clave para la inclusión. Gracias al movimiento de inclusión educativa, la gran mayoría de estudiantes con trastornos del neurodesarrollo están escolarizados en centros ordinarios, mientras que la escolarización en centros específicos es cada vez menos frecuente.

Vemos, por tanto, que el papel del profesorado es clave en la inclusión de estos alumnos, y en especial el grado de conocimientos que estos posean, ya que de ello depende que se realice una identificación y posterior diagnóstico temprano, además de una adecuada intervención, teniendo en cuenta las necesidades de cada individuo.

El objetivo del presente trabajo es realizar una revisión teórica de los estudios que analizan el grado de conocimientos tanto de los maestros como de los futuros maestros respecto a los trastornos del neurodesarrollo, y en concreto respecto a las DEA y el TEA, ya que en los últimos años se han realizado una gran cantidad de estudios que analizan los conocimientos de los maestros, y también de los futuros maestros, en relación al TDAH.

La literatura científica que analiza el grado de conocimientos de los maestros respecto al TDAH reporta que el grado de conocimientos es bajo (López-López, LópezLafuente, Eirís-Puñal, Mulas, y Cardo, 2018). En algunos estudios, se obtiene que si 
bien es cierto que los docentes suelen conocer los criterios diagnósticos y características esenciales del trastorno, carecen de tener el conocimiento suficiente para aplicar las diferentes técnicas de intervención (Topkin, Roman, y Mwaba, 2015).

En cuanto a las DEA y el TEA, los estudios realizados son escasos en comparación al TDAH, por lo que en este trabajo se incluye una revisión teórica que aúna los trabajos empíricos publicados en los últimos cinco años (2014-2018) en relación a estos dos diagnósticos.

\section{Método}

Para realizar la selección de los artículos se han tenido en cuenta los siguientes criterios de inclusión: artículos empíricos publicados en los últimos 5 años (2014-2018) que analizan el grado de conocimientos de los maestros y futuros maestros sobre el TEA y las DEA, y que hayan sido publicados en español e inglés.

Se han utilizado dos bases de datos, en las que se ha realizado la búsqueda: Psyclnfo y Google Scholar. La selección de los artículos se ha realizado a partir de las palabras clave: autism spectrum disorder, ASD, disortography, dyscalculia, dyslexia, learning disabilities, teachers' knowledge. Además, se revisó la lista de referencias bibliográficas de los estudios seleccionados y se revisaron los resúmenes de los estudios obtenidos en las búsquedas.

En cuanto a los criterios de exclusión, se eliminaron de la búsqueda los estudios de revisión, además de aquellos estudios que no hacían uso de un cuestionario de evaluación.

A partir de las palabras clave mencionadas anteriormente, se realizó una búsqueda bibliográfica en las diferentes bases de datos, y considerando los criterios de inclusión y exclusión mencionados, se han seleccionado un total de 10 artículos: 5 sobre DEA y 5 sobre TEA.

\section{Resultados}

En la tabla 1 se incluye toda la información sobre los diez artículos seleccionados. Esta tabla incluye: autores del estudio y año de publicación, país, descripción de los participantes, diagnóstico que analiza cada estudio, instrumento de evaluación utilizado, así como resultados obtenidos. 


\begin{tabular}{|c|c|c|c|c|c|}
\hline $\begin{array}{l}\text { Autorles } \\
\text { (año) }\end{array}$ & $\begin{array}{l}\text { País del } \\
\text { estudio }\end{array}$ & Participantes & Diagnóstico & Instrumento & Resultados \\
\hline \multirow{2}{*}{$\begin{array}{l}\text { Bjornsson, } \\
\text { Saemundsen y } \\
\text { Njardvik } \\
\text { (2018) }\end{array}$} & \multirow[t]{2}{*}{ Islandia } & \multirow{2}{*}{$\begin{array}{l}863 \text { maestros (de Educación } \\
\text { primaria) } \\
-743 \text { maestros generalistas, } \\
\text { - } 105 \text { de educación especial } \\
\text { - } 14 \text { instructores que no disponían de una } \\
\text { certificación reglada. }\end{array}$} & \multirow[t]{2}{*}{ TEA } & \multirow[b]{2}{*}{$\begin{array}{l}\text { Cuestionario de } 41 \text { ítems elaborado por los } \\
\text { autores, y que fue administrado de manera } \\
\text { online. Tres partes: (1) } 13 \text { preguntas } \\
\text { demográficas tales como género, edad, } \\
\text { educación y la ubicación de la escuela donde } \\
\text { los maestros enseñaron; (2) } 19 \text { verdadero- } \\
\text { falso preguntas sobre TEA; y (3) } 9 \text { preguntas } \\
\text { acuerdo-desacuerdo relacionadas con las } \\
\text { opiniones de los encuestados sobre la } \\
\text { calidad de la educación de los niños con } \\
\text { TEA. }\end{array}$} & $\begin{array}{l}\text { Buen conocimiento acerca de los métodos efectivos de enseñanza pero } \\
\text { mayor incertidumbre respecto a la naturaleza de los TEA. }\end{array}$ \\
\hline & & & & & $\begin{array}{l}\text { Los puntos de vista de los docentes sobre la idoneidad de las escuelas } \\
\text { especiales para niños con TEA difirieron, pero la mayoría estuvo de acuerdo } \\
\text { en que su propia educación no cubría adecuadamente los problemas de la } \\
\text { enseñanza de niños con TEA. }\end{array}$ \\
\hline \multirow[t]{2}{*}{ Ghimire (2017) } & \multirow[t]{2}{*}{ Nepal } & \multirow[t]{2}{*}{$\begin{array}{l}150 \text { maestros (de } 16 \text { escuelas de } \\
\text { educación primaria) }\end{array}$} & \multirow[t]{2}{*}{ DEA - general } & \multirow[t]{2}{*}{$\begin{array}{l}\text { Cuestionario elaborado por los autores para } \\
\text { evaluar los conocimientos de los maestros } \\
\text { sobre las DEA. }\end{array}$} & $\begin{array}{l}\text { El } 52.67 \% \text { de los maestros obtuvieron un conocimiento moderadamente } \\
\text { adecuado, mientras que un } 47.33 \% \text { obtuvo un conocimiento inadecuado } \\
\text { sobre las DEA. }\end{array}$ \\
\hline & & & & & $\begin{array}{l}\text { Hubo una asociación significativa entre el grado de conocimientos de los } \\
\text { maestros y el curso en el que estaban impartiendo docencia (mayores } \\
\text { conocimientos en los cursos intermedios de educación primaria). }\end{array}$ \\
\hline $\begin{array}{l}\text { Liu et al. } \\
\text { (2016) }\end{array}$ & China & $\begin{array}{l}471 \text { maestros (de Educación infantil, } \\
\text { trabajando en tres tipos de escuela: } \\
\text { escuelas de provincia, de ciudad, y } \\
\text { de distrito) }\end{array}$ & TEA & $\begin{array}{l}\text { Cuestionario elaborado por los autores, en } \\
\text { base a otros estudios. } 17 \text { ítems sobre } \\
\text { conocimientos del trastorno con tres opciones } \\
\text { de respuesta: verdadero/falso/no sé. Tanto } \\
\text { las respuestas incorrectas como las "no sé" } \\
\text { se puntuaron del mismo modo, con } 0 \text { puntos. }\end{array}$ & $\begin{array}{l}\text { El } 83 \% \text { de los maestros proporcionaron respuestas inexactas a más de la } \\
\text { mitad de los ítems. Se obtuvo que el conocimiento de TEA estaba asociado } \\
\text { con la región geográfica, el nivel de educación de los maestros (mejor cuanto } \\
\text { más superior), y el tipo de escuela (mejor en grandes ciudades). La mayoría } \\
\text { desconocía las asociaciones específicas de TEA y los enfoques de } \\
\text { intervención validados empíricamente. }\end{array}$ \\
\hline \multirow{3}{*}{$\begin{array}{l}\text { Rakap, } \\
\text { Balikci, Parlak- } \\
\text { Rakap, y } \\
\text { Kalkan (2016) }\end{array}$} & \multirow[t]{3}{*}{ Turquía } & \multirow{3}{*}{$\begin{array}{l}504 \text { futuros maestros de cinco } \\
\text { universidades diferentes. } \\
-146 \text { Educación Infantil. } \\
-130 \text { Educación Primaria. } \\
-125 \text { Educación Especial. } \\
-103 \text { de orientación educativa. }\end{array}$} & \multirow[t]{3}{*}{ TEA } & \multirow{3}{*}{$\begin{array}{l}\text { Adaptación del cuestionario sobre } \\
\text { conocimientos del TEA elaborado por } \\
\text { Mavropoulou y Padeliadu (2000). }\end{array}$} & $\begin{array}{l}\text { Los futuros maestros obtuvieron un conocimiento sobre el TEA bajo, } \\
\text { independientemente de la especialidad y la universidad de origen. }\end{array}$ \\
\hline & & & & & $\begin{array}{l}\text { Entre los resultados más sorprendentes se obtuvo que entre el } 10-19 \% \text { de } \\
\text { los participantes informaron falta de capacidad de respuesta materna, y entre } \\
\text { el } 8-17 \% \text { reportaron problemas sociales como las principales causas de } \\
\text { autismo. }\end{array}$ \\
\hline & & & & & $\begin{array}{l}\text { El } 45 \% \text { de los especialistas de educación especial no sabía que el autismo } \\
\text { se diagnostica a través de evaluaciones comportamentales, y un } 37 \% \text { no } \\
\text { sabía que la edad de inicio es de } 3 \text { años o más joven. }\end{array}$ \\
\hline
\end{tabular}




\begin{tabular}{|c|c|c|c|c|c|}
\hline \multirow{3}{*}{$\begin{array}{l}\text { Sanz-Cervera, } \\
\text { Fernández- } \\
\text { Andrés, } \\
\text { Pastor- } \\
\text { Cerezuela y } \\
\text { Tárraga- } \\
\text { Mínguez } \\
\text { (2017) }\end{array}$} & \multirow[t]{3}{*}{ España } & $\begin{array}{l}866 \text { futuros maestros: } 435 \text { de } \\
\text { primero y } 431 \text { de cuarto curso de } \\
\text { magisterio de la Universidad de }\end{array}$ & \multirow[t]{3}{*}{ TEA } & \multirow{3}{*}{$\begin{array}{l}\text { Adaptación del Autism Knowledge } \\
\text { Questionnaire (Haimour y Obaidat, 2013): } \\
\text { Incluye } 30 \text { ítems sobre conocimientos de } \\
\text { características de los estudiantes con TEA. } 3 \\
\text { opciones de respuesta: verdadero/falso/no } \\
\text { sé. Se consideran: conocimientos } \\
\text { (respuestas correctas)/concepciones } \\
\text { erróneas (respuestas incorrectas)/lagunas } \\
\text { ("no sé"). }\end{array}$} & $\begin{array}{l}\text { Los futuros maestros de cuarto obtuvieron un mayor conocimiento y menor } \\
\text { número de lagunas que los alumnos de primero, pero también obtuvieron } \\
\text { más concepciones erróneas. }\end{array}$ \\
\hline & & $\begin{array}{l}\text { Valencia. } \\
\text { De los } 435 \text { de primero: } 162 \text { de } \\
\text { educación infantil (EI) y } 273 \text { de } \\
\text { educación primaria (EP). }\end{array}$ & & & $\begin{array}{l}\text { En cuarto curso, los especialistas en educación especial obtuvieron un } \\
\text { mayor conocimiento y menos lagunas que el resto de futuros maestros } \\
\text { generalistas. Los futuros maestros de PT obtuvieron significativamente un } \\
\text { menor número de concepciones erróneas que los de El y AL. }\end{array}$ \\
\hline & & $\begin{array}{l}\text { De lo } 431 \text { de cuarto: } 229 \text { de El y } 202 \\
\text { de EP (de los cuales } 38 \text { cursaban la } \\
\text { mención de pedagogía terapéutica, } \\
\text { PT, y } 37 \text { la mención de audición y } \\
\text { lenguaje, AL). }\end{array}$ & & & $\begin{array}{l}\text { Haber recibido una formación específica en TEA más allá de la formación } \\
\text { universitaria, y haber tenido contacto con estos niños influye en el grado de } \\
\text { conocimiento, concepciones erróneas y lagunas. }\end{array}$ \\
\hline \multirow[t]{3}{*}{$\begin{array}{l}\text { Shetty y Rai } \\
(2014 a)\end{array}$} & \multirow[t]{3}{*}{ India } & \multirow[t]{3}{*}{$\begin{array}{l}326 \text { maestros (de } 32 \text { escuelas de } \\
\text { educación primaria) }\end{array}$} & \multirow[t]{3}{*}{ TEA } & \multirow{3}{*}{$\begin{array}{l}\text { Cuestionario elaborado por los autores, que } \\
\text { incluye preguntas en relación a los síntomas } \\
\text { característicos del TEA. Dos opciones de } \\
\text { respuesta: Verdadero o falso. }\end{array}$} & $\begin{array}{l}\text { El } 95.7 \% \text { de los maestros eran conscientes de la existencia del autismo, pero } \\
\text { solo el } 21 \% \text { poseía un conocimiento adecuado. }\end{array}$ \\
\hline & & & & & $\begin{array}{l}\text { De lo } 326 \text {, tan solo } 71 \text { profesores habían tenido un entrenamiento previo en } \\
\text { TEA. }\end{array}$ \\
\hline & & & & & $\begin{array}{l}\text { Los docentes con más experiencia y capacitación previa tuvieron un mejor } \\
\text { desempeño. }\end{array}$ \\
\hline \multirow{3}{*}{$\begin{array}{l}\text { Shetty y Rai } \\
(2014 b)\end{array}$} & \multirow[t]{3}{*}{ India } & \multirow{3}{*}{$\begin{array}{l}314 \text { maestros (de } 32 \text { escuelas de } \\
\text { educación primaria) }\end{array}$} & \multirow[t]{3}{*}{ DEA - dislexia } & \multirow{3}{*}{$\begin{array}{l}\text { Cuestionario elaborado por los autores que } \\
\text { incluye } 12 \text { ítems sobre señales de alerta y } \\
\text { síntomas de dislexia. }\end{array}$} & 1 de cada 3 maestros obtuvo un conocimiento adecuado de la dislexia. \\
\hline & & & & & $\begin{array}{l}\text { De los } 314 \text { participantes, } 262 \text { conocían el término de dislexia, y tan solo } 24 \\
\text { habían recibido entrenamiento previo. }\end{array}$ \\
\hline & & & & & $\begin{array}{l}\text { La experiencia docente y un entrenamiento previo correlacionaron } \\
\text { positivamente con el grado de conocimientos. }\end{array}$ \\
\hline $\begin{array}{l}\text { Shukla y } \\
\text { Agrawal } \\
(2015)\end{array}$ & India & $\begin{array}{l}68 \text { maestros (de } 15 \text { escuelas de } \\
\text { educación primaria) }\end{array}$ & DEA - general & $\begin{array}{l}\text { Cuestionario elaborado por los autores que } \\
\text { evalúa el grado de conocimientos sobre DEA } \\
\text { por parte de los maestros. }\end{array}$ & $\begin{array}{l}\text { Se obtuvo un bajo nivel de conocimientos sobre DEA por parte de los } \\
\text { maestros: } \\
\text { - El } 11 \% \text { obtuvo un nivel de conocimientos satisfactorio. } \\
\text { - El } 20 \% \text { obtuvo un nivel de conocimientos pobre. } \\
\text { - El } 67 \% \text { no tenía conocimientos sobre DEA. }\end{array}$ \\
\hline \multirow{3}{*}{$\begin{array}{l}\text { Soriano- } \\
\text { Ferrer, } \\
\text { Echegaray- } \\
\text { Bengoa, y } \\
\text { Joshi (2016) }\end{array}$} & \multirow[t]{3}{*}{$\begin{array}{l}\text { España y } \\
\text { Perú }\end{array}$} & \multirow[t]{3}{*}{$\begin{array}{l}267 \text { maestros y } 246 \text { futuros } \\
\text { maestros }\end{array}$} & \multirow[t]{3}{*}{ DEA - dislexia } & \multirow{3}{*}{$\begin{array}{l}\text { The Knowledge and Beliefs about } \\
\text { Developmental Dyslexia Scale (KBDDS): } \\
\text { Evalúa Conocimientos, concepciones } \\
\text { erróneas y lagunas sobre dislexia. Incluye } 36 \\
\text { items sobre sintomatología diagnóstico y } \\
\text { tratamiento, y tiene tres opciones de } \\
\text { respuesta: verdadero/falso/no sé. }\end{array}$} & $\begin{array}{l}\text { Los maestros en activo obtuvieron puntuaciones significativamente más altas } \\
\text { en la escala total, y en las subescalas de síntomas/diagnóstico e información } \\
\text { general, que los futuros maestros. }\end{array}$ \\
\hline & & & & & $\begin{array}{l}\text { Los porcentajes de concepciones erróneas y lagunas fueron mayores para } \\
\text { los futuros maestros que para los maestros en activo. }\end{array}$ \\
\hline & & & & & $\begin{array}{l}\text { La autoeficacia percibida de los docentes en activo, los años de experiencia, } \\
\text { haber realizado un posgrado en dislexia y la exposición previa a un niño con } \\
\text { dislexia se relacionaron positivamente con el conocimiento sobre la dislexia. }\end{array}$ \\
\hline
\end{tabular}




\begin{tabular}{|c|c|c|c|c|c|}
\hline \multirow[t]{2}{*}{$\begin{array}{l}\text { Washburn, } \\
\text { Mulcahy, } \\
\text { Musante y } \\
\text { Joshi (2017) }\end{array}$} & \multirow[t]{2}{*}{ EEUU } & $\begin{array}{l}271 \text { maestros de } 8 \text { universidades } \\
\text { diferentes: } 148 \text { maestros } \\
\text { generalistas, y } 123 \text { especialistas de } \\
\text { educación especial }\end{array}$ & \multirow[t]{2}{*}{$\begin{array}{l}\text { DEA - dislexia y } \\
\text { dificultades en } \\
\text { comprensión } \\
\text { lectora }\end{array}$} & \multirow[t]{2}{*}{$\begin{array}{l}\text { Adaptación del cuestionario elaborado por } \\
\text { Washburn, Joshi y Cantrell (2011) para } \\
\text { evaluar conocimientos sobre DEA. }\end{array}$} & $\begin{array}{l}\text { Los futuros maestros obtuvieron un grado de conocimientos adecuado } \\
\text { acerca de las dificultades en comprensión lectora, pero obtuvieron un grado } \\
\text { elevado de concepciones erróneas acerca de la dislexia. }\end{array}$ \\
\hline & & $\begin{array}{l}\text { De los } 271,229 \text { no tenían } \\
\text { experiencia docente, } 26 \text { tenían una } \\
\text { experiencia entre } 1-2 \text { años, y } 16 \\
\text { tenían entre 3-5 años de } \\
\text { experiencia. }\end{array}$ & & & $\begin{array}{l}\text { El nivel de experiencia y el hecho de leer acerca de las DEA no predijeron un } \\
\text { mayor grado de conocimientos. No obstante, el nivel de experiencia sí } \\
\text { predijo el grado de concepciones erróneas sobre la dislexia. }\end{array}$ \\
\hline
\end{tabular}


Los 10 artículos seleccionados evalúan los conocimientos, ya sea de los maestros o futuro maestros, en relación al TEA o a las DEA. En el caso de las DEA, de los 5 artículos seleccionados, dos evalúan los conocimientos de los maestros en relación a las dificultades de aprendizaje a nivel general (Ghimire, 2017; Shukla y Agrawal, 2015); dos hacen referencia a los conocimientos en relación a la dislexia (Shetty y Rai, 2014; Shetty y Rai, 2014b); y uno incluye conocimientos tanto sobre dislexia como dificultades en la comprensión lectora (Washburn et al., 2017). También se realizó una búsqueda respecto a los conocimientos de los maestros sobre las dificultades en escritura y en matemáticas, ya que estas también forman parte de la etiqueta diagnóstica de las DEA, pero no se encontraron resultados al respecto.

En cuanto a los estudios realizados con maestros o futuros maestros, en el caso del TEA, tres de los estudios seleccionados se realizaron con maestros en activo (Bjornsson et al., 2018; Liu et al., 2016; Shetty y Rai, 2014a), y dos se realizaron con futuros maestros, estudiantes de magisterio (Rakap et al., 2016; Sanz-Cervera et al., 2017). Con respecto a las DEA, tres de los estudios también se realizaron únicamente con maestros en activo (Ghimire, 2017; Shetty y Rai, 2014b; Shukla y Agrawal, 2015), mientras que los otros dos se realizaron tanto con maestros en activo como con futuros maestros de manera conjunta (Soriano-Ferrer et al., 2016; Washburn, 2017).

En todos los estudios seleccionados, se hizo o bien una adaptación de cuestionarios utilizados en estudios anteriores, o incluso en algunos casos, los autores elaboraron un cuestionario para analizar los aspectos que consideraron conveniente. En todos los casos, se realizaron los estudios psicométricos pertinentes.

Como se puede comprobar en la tabla 1, cada estudio se realizó en un país diferente, y con independencia del contexto, en todos los casos se obtuvo que tanto los maestros como los futuros maestros poseen un conocimiento bajo en relación al TEA y también a las DEA.

Al comparar el nivel de conocimientos de maestros y futuros maestros, los estudios que comparan dicho aspecto obtuvieron resultados dispares. En el caso del estudio de Soriano-Ferrer et al. (2016), se obtuvo que los maestros en activo poseen un nivel de conocimientos superior que los futuros maestros en relación a la dislexia, además de que los porcentajes de concepciones erróneas y lagunas fueron mayores para los futuros maestros que para los maestros en activo. En el estudio de Washburn et al. (2017), sin embargo, se obtuvo que el nivel de experiencia no predecía un mayor grado de conocimientos en relación a la dislexia, aunque sí lo hacía en el caso de las concepciones erróneas. Generalmente, en los estudios con maestros en activo, los docentes con más experiencia y capacitación suelen obtener un mejor desempeño (Shetty y Rai, 2014a, 2014b; Soriano-Ferrer et al., 2016).

Respecto a los estudios con futuros maestros, todos reportan un conocimiento bajo de los estudiantes en relación a los trastornos del neurodesarrollo estudiados con independencia del contexto. De hecho, en el estudio de Rakap et al. (2016), realizado en cinco universidades diferentes, se obtuvo un conocimiento sobre el TEA bajo, independientemente de la especialidad y la universidad de origen.

En el estudio de Sanz-Cervera et al. (2017), se realizó un análisis con mayor profundidad, analizando el grado de conocimientos de los futuros maestros sobre el TEA, tanto en su primer año como en su último año de magisterio, además de realizar un análisis por especialidades. En este estudio, los futuros maestros de cuarto obtuvieron un mayor conocimiento y menor número de lagunas que los alumnos de primero, aunque también obtuvieron más concepciones erróneas. Los especialistas en 
educación especial obtuvieron un mayor conocimiento y menos lagunas que el resto de futuros maestros generalistas; y los futuros maestros de PT obtuvieron significativamente un menor número de concepciones erróneas que los de AL. Se obtuvo que el hecho de haber recibido una formación específica en TEA más allá de la formación universitaria, y haber tenido contacto con estos niños influye en el grado de conocimiento, concepciones erróneas y lagunas.

En la misma línea, en el caso de la dislexia, en el estudio de Soriano-Ferrer et al. (2016), también se obtuvo que los años de experiencia, haber realizado un posgrado en dislexia y la exposición previa a un niño con dislexia se relacionaron positivamente con el grado de conocimientos sobre la dislexia de los maestros.

\section{Discusión}

Los resultados obtenidos, en línea con la literatura previa, ponen de manifiesto que existen carencias en la formación del profesorado en cuanto a los conocimientos que poseen sobre los trastornos del neurodesarrollo.

Aunque, en los pocos estudios en lo que se ha realizado una comparación entre los conocimientos de los maestros en activo y de los futuros maestros, se obtiene que los maestros en activo poseen un mayor grado de conocimientos (Soriano-Ferrer et al., 2016), parece ser que estos resultados no son concluyentes (Washburn et al., 2017).

Algunos de los estudios que analizan posibles factores relacionados con el grado de conocimientos, apuntan que estos pueden estar moderados por el tipo de formación e intensidad de la misma que recibe el profesorado a lo largo tanto de su formación inicial como de su formación continua, y también en relación al grado de experiencia docente o de contacto que los estudiantes hayan podido tener con alumnos con este tipo de trastornos (Ghimire, 2017; Liu et al., 2016; Shetty y Rai, 2014a, 2014b; Soriano-Ferrer et al., 2016); un aspecto que influye no únicamente en el grado de conocimientos, sino también en relación al número de posibles concepciones erróneas por parte de los maestros (Washburn et al., 2017).

Algunos estudios que han analizado el impacto de la formación en relación al grado de conocimientos, han obtenido que se produce un incremento significativo tras recibir un entrenamiento (Giannopoulou, Pasalari, Korkoliakou, y Douzenis, 2018), aunque se trate de una formación limitada (Leblanc, Richardson y Burns, 2009). En concreto, se ha evidenciado que la tecnología puede ser un medio interesante que permite incrementar los conocimientos de los maestros de un modo ameno y divertido, además de ser instructivo (Hart y More, 2013).

Al analizar posibles diferencias entre los conocimientos de los maestros generalistas y de los maestros en educación especial, únicamente encontramos un estudio que analiza dicho aspecto, e incluye futuros maestros. En este estudio, se obtuvo que si bien los maestros especialistas en educación especial poseen un mayor grado de conocimientos y menos lagunas que el resto de futuros maestros generalistas, también cabe considerar diferencias entre la formación de los especialistas, ya que los futuros maestros de $\mathrm{PT}$ obtuvieron significativamente un menor número de concepciones erróneas que los de AL (Sanz-Cervera et al., 2017).

Este fue un resultado sorprendente, ya que ambas menciones incluían la misma carga lectiva sobre TEA a lo largo de la titulación. No obstante, este aspecto puede ser debido a que la formación de los maestros de AL suele ser más específica sobre los posibles problemas de comunicación que los niños con TEA suelen presentar, 
mientras que la formación de los maestros de PT suele ser más general, considerando todas las áreas de intervención que los estudiantes con TEA necesitan trabajar.

Teniendo en cuenta la repercusión que puede ocasionar el grado de conocimientos que los maestros posean sobre los trastornos del neurodesarrollo y considerando el bajo nivel de conocimientos obtenido en los diferentes estudios, es evidente que la formación es un aspecto clave. Revisiones recientes en nuestro país, en cambio, muestran que la formación inicial es escasa (Vélez-Clavo, Tárraga-Mínguez, Fernández-Andrés, y Sanz-Cervera, 2017). A pesar de que la relativamente reciente reforma de los planes de estudio fue una buena oportunidad para mejorar la situación anterior (Guerrero, 2011), parece ser que en realidad se ha producido un decremento en el número de créditos en materia de educación especial.

Estos hallazgos ponen en evidencia que se necesita una mayor capacitación e instrucción docente. Generalmente, los maestros informan que están dispuestos y motivados para adquirir las habilidades necesarias para maximizar las experiencias educativas de sus alumnos (Liu et al. 2016), por lo que es cuestión de realizar un análisis de los planes de estudio en el caso de la formación inicial, y una análisis de los programas de formación continua en el caso de los maestros en activo.

Es evidente que los futuros planes de estudio del Grado de Maestro deberían incluir un mayor número de créditos en materia de educación especial; una formación, que no debería dirigirse únicamente a la presentación teórica de conocimientos, sino que debería enfatizar en la importancia de la detección y una mayor formación en estrategias de intervención, además de poseer una mayor concienciación sobre la importancia de las actitudes que los mismos docentes transmiten a los niños, y el hecho de llevar a cabo un trabajo cooperativo entre la escuela, la familia, los especialistas y otros servicios comunitarios.

En la presente revisión se han hallado algunas limitaciones, como el reducido número de estudios empíricos que cumplían los criterios de inclusión propuestos, además del uso de diferentes cuestionarios de evaluación, un aspecto que impide poder comparar los resultados obtenidos en los diferentes estudios.

Como futuras líneas de investigación es necesario continuar investigando para profundizar en el análisis de los conocimientos tanto de los maestros como de los futuros maestros en relación a los trastornos del neurodesarrollo, pudiendo así desarrollar programas de formación, tanto inicial como continua, que permitan formar adecuadamente al profesorado. Para ello, es necesario realizar un análisis de los diferentes planes de estudio que ofrecen las universidades y los planes de formación continua del profesorado de las diferentes comunidades.

También sería conveniente realizar más estudios que comparen la formación tanto de maestros generalistas como de maestros especialistas en educación especial, ya que una escuela inclusiva requiere de una formación especializada no solo en el caso de los especialistas, sino también de los generalistas. Sería interesante elaborar cuestionarios estandarizados que analicen el grado de conocimientos de los maestros, ya que ello permitiría obtener una mayor fiabilidad en los resultados.

Además de continuar en la línea de analizar los conocimientos de manera cuantitativa, a través de cuestionarios, también cabría hacer uso de técnicas cualitativas que permitan además saber más sobre las actitudes del profesorado y cómo estas se asocian a los conocimientos. Ello permitiría realizar un trabajo personal mucho más profundo, que engloba no tanto la adquisición de conocimientos a nivel externo, sino 


\section{más bien el hecho de realizar una reprogramación mental sobre todas aquellas creencias obsoletas que todavía se transmiten a nivel social.}

\section{Referencias}

Los artículos incluidos en la revisión están marcados con asterisco $\left({ }^{*}\right):$

American Psychiatric Association (2013). Diagnostic and statistical manual of mental disorders (5th ed). Arlington, VA: American Psychiatric Publishing.

*Bjornsson, B. G., Saemundsen, E., y Njardvik, U. (2018). A survey of Icelandic elementary school teachers' knowledge and views of autism-Implications for educational practices. Nordic Psychology.

Canals, J., Morales-Hidalgo, P., Claustre-Jané, M. y DomènechLlaberia, E. (2016). ADHD Prevalence in Spanish Preschoolers: Comorbidity, Socio-Demographic Factors, and Functional Consequences. Journal of Attention Disorders.

Catalá-López, F., Peiro, S., Ridao, M., Sanfélix-Gimeno, G., Gènova-Maleras, R., y Catalá, M. A. (2012). Prevalence of attention deficit hyperactivity disorder among children and adolescents in Spain: A systematic review and meta-analysis of epidemiological studies. BMC Psychiatry. 12:168.

*Ghimire, S. (2017). Knowledge of primary school teacher regarding learning disabilities in school children. Journal of Nobel Medical College, 6(1), 29-35.

Giannopoulou, I., Pasalari, E., Korkoliakou, P., y Douzenis, A. (2018). Raising Autism Awareness among Greek Teachers. International Journal of Disability, Development and Education.

Guerrero, M. J. L. (2011). La situación de la formación en educación inclusiva en los nuevos títulos de grado de maestro en España. Revista Interuniversitaria de Formación del Profesorado, 70(25,1), 145-163.

Hart, J. E., y More, C. M. (2013). Investigating the impact of technology on pre-service teacher knowledge of autism spectrum disorder. Education and Training in Autism and Developmental Disabilities, 504-513

Leblanc, L., Richardson, W., y Burns, K. A. (2009). Autism spectrum disorder and the inclusive classroom: Effective training to enhance knowledge of ASD and evidence-based practices. Teacher Education and Special Education, 32(2), 166-179.

*Liu, Y., Li, J., Zheng, Q., Zaroff, C. M., Hall, B. J., Li, X., y Hao, Y. (2016). Knowledge, attitudes, and perceptions of autism spectrum disorder in a stratified sampling of preschool teachers in China. BMC Psychiatry, 16:142.

López-López, A., López-Lafuente, A., Eirís-Puñal, J., Mulas, F., y Cardo, E. (2018). Estudio de los conocimientos de los maestros de educación primaria sobre el trastorno por déficit de atención/hiperactividad. Revista de Neurología, 66(Supl 1), s121-6.
Mavropoulou, S., y Padeliadu, S. (2000). Greek teachers' perceptions of autism and implications for educational practice: A preliminary analysis. Autism, 4(2), 173-183.

Morales-Hidalgo, P., Roigé-Castellví, J., Hernández-Martínez, C., Voltas, N., y Canals, J. (2018). Prevalence and Characteristics of Autism Spectrum Disorder Among Spanish School-Age Children. Journal of Autism and Developmental Disorders, 48(9), 3176-3190.

*Rakap, S., Balikci, S., Parlak-Rakap, A., y Kalkan, S. (2016). An analysis of Turkish pre-service teachers' knowledge of autism spectrum disorder: Implications for teacher preparation programs. SAGE Open, 6(3)

*Sanz-Cervera, P., Fernández-Andrés, M. I., Pastor-Cerezuela, G., y Tárraga-Mínguez, R. (2017). Pre-Service Teachers' Knowledge, Misconceptions and Gaps About Autism Spectrum Disorder. Teacher Education and Special Education, 40(3), 212-224

*Shetty, A., y Rai, B. S. (2014a). Awareness and Knowledge of Autism Spectrum Disorders among Primary School Teachers in India. International Journal of Health Sciences and Research, 4(4), 80-85.

*Shetty, A., y Rai, B. S. (2014b). Awareness and knowledge of dyslexia among elementary school teachers in India. Journal Of Medical Science And Clinical Research, 2(5), 1135-1143.

*Shukla, P., y Agrawal, G. (2015). Awareness of learning disabilities among teachers of primary schools. Online Journal of Multidisciplinary Research, 1(1), 33-38.

*Soriano-Ferrer, M., Echegaray-Bengoa, J., y Joshi, R. M. (2016). Knowledge and beliefs about developmental dyslexia in preservice and in-service Spanish-speaking teachers. Annals of Dyslexia, 66(1), 91-110.

Soriano-Ferrer, M., y Morte-Soriano, M. R. (2017). Developmental Dyslexia in Spain. In Learning Disabilities-An International Perspective. Ed. InTech. doi: 10.5772/in tech open.69009

Topkin, B., Roman, N. V., y Mwaba, K. (2015). Attention Deficit Disorder (ADHD): Primary School Teachers' Knowledge of Symptoms, Treatment and Managing Classroom Behaviour. South African Journal of Education, 35(2), 988.

Vélez-Clavo, X., Tárraga-Mínguez, R., Fernández-Andrés, M. I., y Sanz-Cervera, P. (2017). Formación inicial de maestros en Educación Inclusiva: una comparación entre Ecuador y España. Revista de Educación Inclusiva, 9(3), 75-94.

Washburn, E. K., Joshi, R. M., y Cantrell, E. B. (2011). Are preservice teachers prepared to teach struggling readers? Annals of Dyslexia, 61, 21-43.

*Washburn, E. K., Mulcahy, C. A., Musante, G., y Joshi, R. (2017). Novice Teachers' Knowledge of Reading-Related Disabilities and Dyslexia. Learning Disabilities: A Contemporary Journal, 15(2), 169-191. 